\title{
Effects of longitudinal asymmetric distribution of a lipid core on plaque wall stress
}

\author{
Pengsrorn CHHAI*, Jin Hyun LEE* and Kyehan RHEE* \\ *Department of Mechanical Engineering, Myongji University \\ 38-2 Namdong, Cheoin-gu, Yongin, Gyeonggi-do 449-728, Republic of Korea \\ E-mail: khanrhee@mju.ac.kr
}

Received: 31 October 2016; Revised: 13 December 2016; Accepted: 26 January 2017

\begin{abstract}
The rupture of the atherosclerotic plaque is related to the mechanical stress and structural integrity of plaque wall tissues. In order to investigate the longitudinal asymmetry across the stenosis of the arterial plaque wall, asymmetric plaque wall models were constructed by skewing the lipid core distribution in the upstream direction. Wall stress and blood flow in the coronary artery models were computationally analyzed considering fluid and structure interaction. The values of maximum cap stress increased, and its location moved toward the proximal cap as asymmetry increased. Hemodynamic wall shear stress (WSS) did not change much owing to negligible changes in luminal geometry, but the maximum WSS and the spatial gradient of WSS were higher in the asymmetry models than in the symmetry model. The pressure drop and pressure gradient across the stenosis were also higher in the asymmetry models. Because higher peak wall stress, wall strain, increased WSS, WSS gradient, pressure drop, and pressure gradient are correlated with weakening and rupture of the plaque wall, we suspect that longitudinal asymmetric distribution of the lipid core in the plaque could affect plaque wall stability and vulnerability.
\end{abstract}

Key words : Plaque rupture, Mechanical stress, Fluid structure interaction, Computational analysis, Plaque wall composition

\section{Introduction}

Atherosclerotic plaque rupture contributes to acute coronary syndrome, one of the leading causes of death in the world. It is composed of lipid rich necrotic cores and calcium deposits enclosed by a protective fibrous cap, which is a thin fibrous tissue between the lumen of the blood vessel and the lipid core. If the fibrous cap is disrupted, a cascade of events that include thrombosis, coronary occlusion, and subsequent myocardial infarction could occur (Libby, 2013). Studies have shown that the mechanical stress and structural integrity of the wall tissues of the plaque determine its susceptibility to disruption.

Two different types of stresses act on the plaque tissues: circumferential tensile wall stress, which is caused by pulsating blood pressure, and luminal wall shear stress, which is caused by blood flow. Because tensional wall stress is several orders of magnitude higher than wall shear stress exerted by blood flow (Salger et al., 2005), plaque rupture has been attributed to circumferential wall stress (Ohayon et al., 2005), which is influenced by the morphology and mechanical properties of the plaque. It has been shown that atherosclerotic plaques with large plaque burdens (defined as the plaque area over the vessel area at the cross section) and thin cap thicknesses (less than $65 \mu \mathrm{m}$ ) are more vulnerable (Burke et al. 1997). Computational stress analysis has been performed in order to estimate the plaque wall stress using the idealized plaque models (Ohayon et al. 2008, Akyildiz et al., 2011, Zareh et al., 2015). Recently, three dimensional patient specific plaque models have been reconstructed from diagnostic medical images such as ultrasound, OCT, and MRI images for stress analysis (Nieuwstadt et al., 2013, Kelly-Arnold et al., 2013, Wang et al., 2015) and computational analysis has been performed. They provide a more realistic geometry for wall stress and flow analyses, but they are not ideally suited for obtaining accurate plaque wall morphology due to limitations that include 
low spatial resolutions, image artifacts and difficulties differentiating plaque tissues and three dimensional volume reconstruction.

Although hemodynamic wall shear stress (WSS) is not high enough to disintegrate the plaque wall, the endothelium is very sensitive to it. Furthermore, it affects the function of the cells and tissues of the intima during disease progression. Intima thickening is correlated with low and oscillatory shear stress (Peiffer et al. 2013), and the plaque region exposed to lower shear stress shows lower strain (Gijsen et al., 2008). In the stenotic lumen observed in the advanced stage of atherosclerotic plaque, the endothelium is exposed to variable hemodynamic stress along the longitudinal direction. In the upstream of the stenosis, elevated wall shear stress activates inflammatory path ways and the infiltration of macrophages (Dirksen et al., 1998) and dendritic cells (Yilmaz et al., 2007) into the region is increased. In contrast, smooth muscle cell proliferations and increased endothelial cell apoptosis are found in the downstream region. Changes in cellular function and the distribution of tissue along the longitudinal direction of the stenosis cause variations in the cross sectional morphology and mechanical properties of the plaque wall. However, the longitudinal heterogeneity along the stenotic plaque wall has not been considered in analyzing mechanical stress in plaque models.

In this study, mechanical stress in the plaque wall and hemodynamic stress in the lumen were computationally analyzed for arterial plaque models considering fluid and structure interaction. Plaque wall composition may be different for the upstream and downstream walls of the throat, where the lumen has minimal cross sectional area. Longitudinal asymmetry of the plaque wall was modeled by skewing the lipid core distribution upstream of the stenosis and increasing the cap tissue thickness downstream of the stenosis, because intimal thickening is more pronounced in the distal wall. Effects of lipid core distribution skewness (asymmetry) on plaque wall stress, strain and hemodynamic stress were explored.

\section{Methods}

An ideal three dimensional coronary artery with an eccentric stenosis was modeled using the 3-D modeler (SolidWorks, Dassault Systèmes, MA, U.S.A.) as shown in Fig. 1. A cylindrical vessel of $100 \mathrm{~mm}$ long was attached in the upstream and downstream of the stenotic plaque model in order to provide flow development as well as to minimize the fixed wall boundary effect on wall deformation. The diameter of the lumen was $3 \mathrm{~mm}$, while the length of the stenosis was $8.2 \mathrm{~mm}$. The stenotic plaque wall is composed of the lipid core and fibrous cap. The luminal height of the throat (minimum lumen cross section) was about $1 \mathrm{~mm}$, and the area reduction was about $36 \%$. The vessel wall thickness was $1 \mathrm{~mm}$, and the minimum thickness of the fibrous cap was about $40 \mu \mathrm{m}$, as shown in Fig. 2 . The symmetry model had a symmetric lipid core with respect to the throat, and the half base length of the lipid core was $3.775 \mathrm{~mm}$ (Sym). The distal base length of the lipid core was decreased to $3.28 \mathrm{~mm}$ (Asym1), $2.78 \mathrm{~mm}$ (Asym2) and $1.81 \mathrm{~mm}$ (Asym3) in the asymmetric models, and the cap thickness was increased in order to construct the same lumen geometry for all plaque vessel models. The Asym3 model had the most asymmetric lipid core distribution.

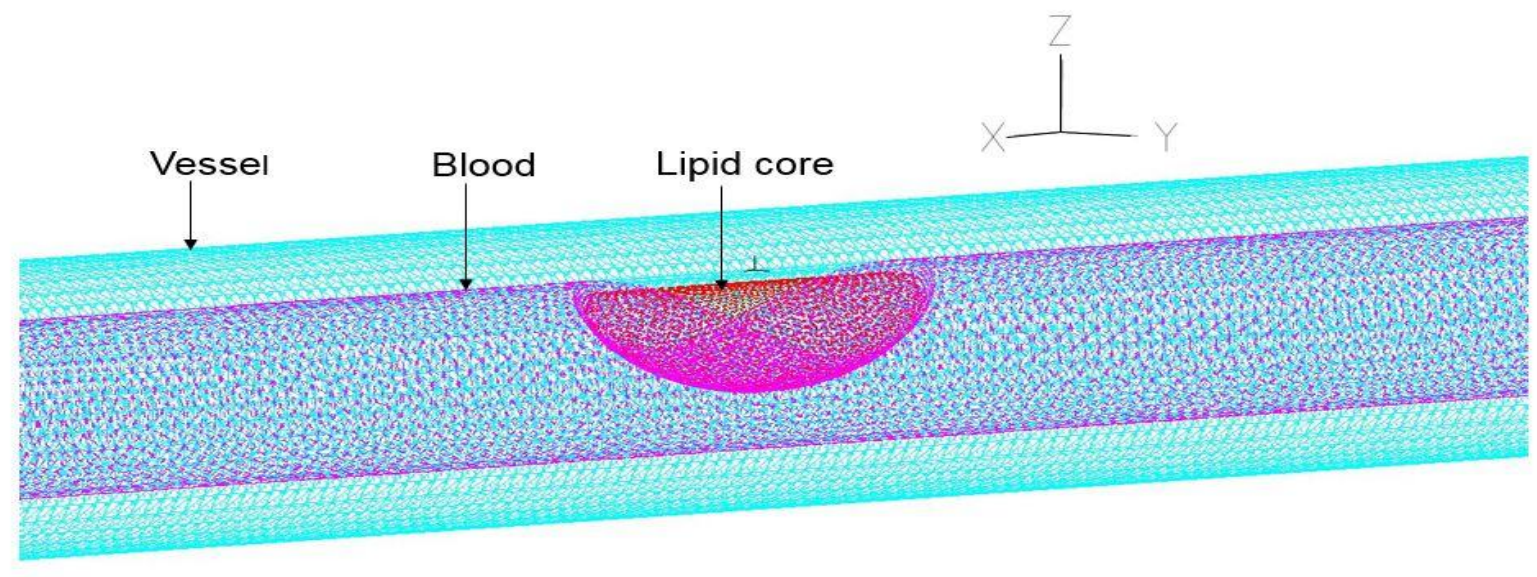

Fig. 1 Three dimensional coronary artery model with an eccentric stenosis and the computational meshes for the symmetric lipid core model. 

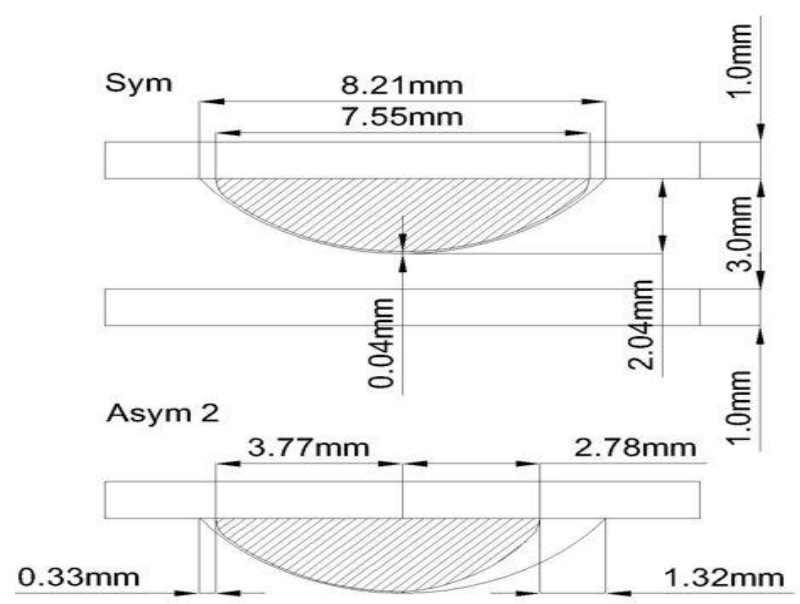

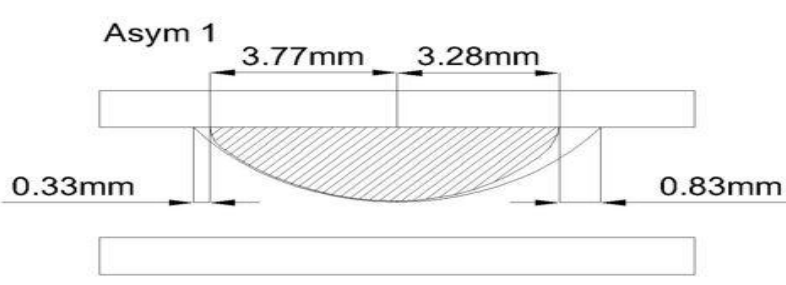

Asym 3

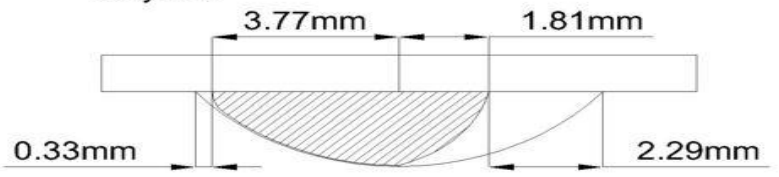

Fig. 2 Geometry of plaque models in the longitudinal cross section (a) Sym, (b) Asym1, (c) Asym2, and (d) Asym3. The symmetry model (Sym) had the half base length of the lipid core was $3.775 \mathrm{~mm}$. The distal base length of the lipid core was $3.28 \mathrm{~mm}$ (Asym1), $2.78 \mathrm{~mm}$ (Asym2) and $1.81 \mathrm{~mm}$ (Asym3) in the asymmetric models.

The constructed three dimensional models were imported into ADINA version 9.2 (ADINA R\&D Inc., MA, U.S.A.), a commercial finite element package, to analyze the stress and flow fields in the plaque models considering fluid and structure interaction (FSI). ADINA uses unstructured finite element methods for both fluid and solid models, and nonlinear incremental iterative procedures were used to handle FSI problems. It has been tested in many FSI problems with hyperelastic materials (Bathe, 1996, 2002), and also has been validated in FSI analysis in arteries and plaques (Yang et al., 2010, Tang et al., 2014, Yuan et al., 2015). The 4-node tetrahedral elements were used for computational meshes, and they were fitted with the shape of the vessel lumen and the plaque wall tissue. Finer meshes were used in the plaque cap and the lipid core zone to accommodate abrupt changes in geometry. Mesh was refined near the fibrous cap to get mesh independent solutions in stress and strain calculations. Mesh analysis was performed by increasing mesh size near the fibrous cap zone until the maximum stress values converged within 5 percent. The mesh was then chosen for FSI computation. In the symmetric model, the vessel and lipid core consisted of about 99,679 and 11,991elements, respectively. In the asymmetric models, about 91,000 to 95,000 elements were used for the vessel and about 5,000 to 9,100 elements were used for lipid core. The fluid domain of all four models consisted of 65,556 elements. For all models, the fluid was assumed to be incompressible and Newtonian, with a density of $1,050 \mathrm{~kg} / \mathrm{m} 3$ and a viscosity of $0.0035 \mathrm{~kg} / \mathrm{m}$.s. No slip boundary conditions were used on the walls. The Navier-Stokes equation with arbitrary Lagrangian-Eulerian formulation was used to solve governing equations in the presence of fluid-structure interaction (FSI), and a transient implicit scheme was applied to fluid flow computations. The velocity waveform obtained from a coronary flow waveform and the physiological pressure waveform (Rambhia et al., 2012) were applied at the inlet and the outlet of the vessel, respectively, in order to consider the phase lag between the pressure and the flow waves (Fig. 3). The inlet velocity was assumed to be uniform, and initial velocities and pressure were assumed to be zero. The computational simulation was performed for three cardiac cycles until solutions became periodic and converged. The computational results from the third period were used for analysis.

The Mooney-Rivlin (M-R) model was used to describe the material properties of the wall of the plaque tissue, which was assumed to be hyperelastic and anisotropic. The modified $\mathrm{M}-\mathrm{R}$ model after adding anisotropic terms is given by

$$
\begin{aligned}
& W=C_{1}\left(I_{1}-3\right)+C_{2}\left(I_{2}-3\right)+D_{1}\left[\exp \left(D_{2}\left(I_{1}-3\right)\right)-1\right]+\frac{K_{1}}{2 K_{2}}\left[\exp \left(K_{2}\left(I_{4}-1\right)^{2}-1\right)\right] \\
& I_{1}=\sum C_{i i} \quad I_{2}=\frac{1}{2}\left(I_{1}^{2}-C_{i j} C_{i j}\right) \quad I_{4}=C_{i j}\left(n_{c}\right)_{i}\left(n_{c}\right)_{j} \\
& C=C_{i j}=F^{T} F \quad F=F_{i j}=\frac{\partial X_{i}}{\partial X_{j}}
\end{aligned}
$$



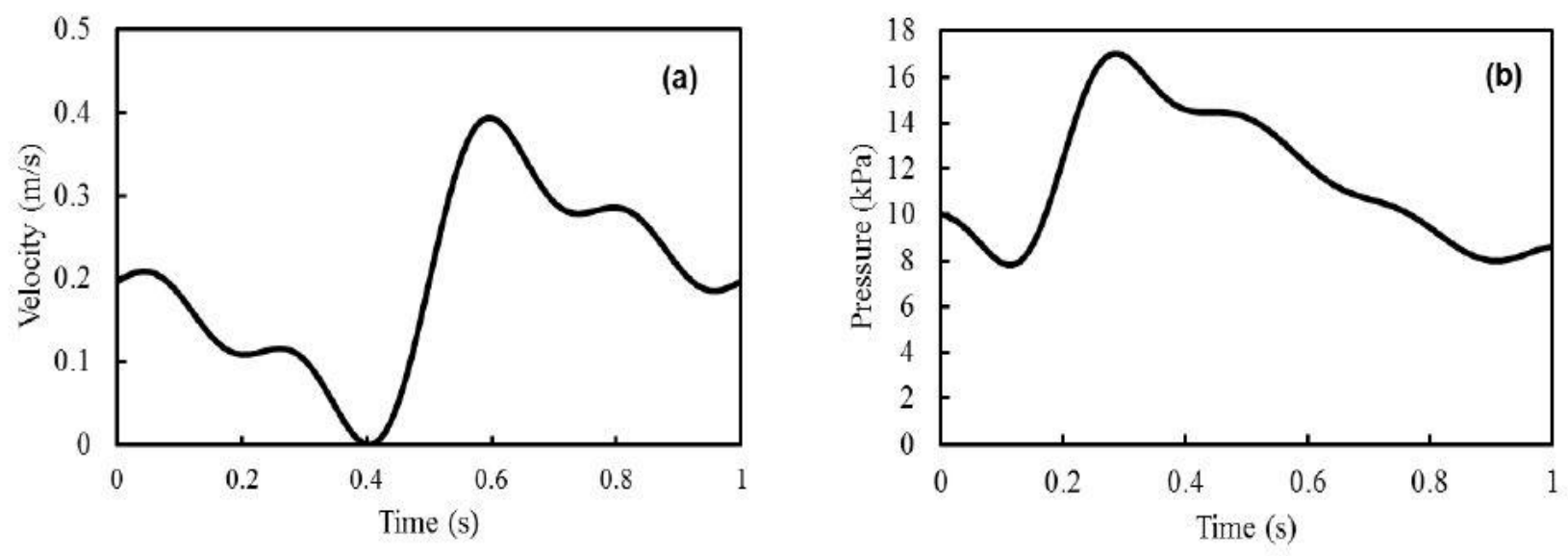

Fig. 3 Velocity (a) and pressure (b) waveforms at the inlet and outlet of the artery models. The phase lag between the pressure and the flow waves in a coronary artery is considered.

where $C=C_{i j}=F^{T} F$ is the right Cauchy-Green deformation tensor, $X_{i}$ is the current position, $X_{j}$ is the original position, $n_{c}$ is the circumferential direction of the vessel, and $I_{l}$ and $I_{2}$ are the first and second strain invariants. The parameters in the strain energy density function, $C_{1}, C_{2}, D_{1}, D_{2}, K_{1}$, and $K_{2}$, were chosen to match the mechanical properties of the plaque wall obtained from experimental measurements. Data from planar biaxial tests of coronary arteries were used (Kural et al., 2012) to fit the modified Mooney-Rivlin model, and the parameters in the strain energy density function were $C_{l}=-1312.9 \mathrm{kPa}, C_{2}=114.7 \mathrm{kPa}, \mathrm{D}_{1}=629.7 \mathrm{kPa}, \mathrm{D}_{2}=2.0, K_{l}=35.9 \mathrm{kPa}, K_{2}=23.5$. The lipid core was assumed to be incompressible and isotropic with $C_{l}=0.5 \mathrm{kPa}, \mathrm{D}_{1}=0.5 \mathrm{kPa}$ and $\mathrm{D}_{2}=0.5$, and $C_{2}$ and $K_{1}$ are zeroes. (Tang et al., 2009). The arterial wall was fixed at the lower edge of the outer vessel wall, and both inlet and outlet cross-sections of the vessel wall were fixed in all directions. FSI boundary conditions were imposed on the luminal surface of the arterial wall.

\section{Results and Discussion}

Equivalent stress in the plaque wall, which is the scalar quantity defined on the surface, was compared for the different plaque models. Figure 4 shows the contour of equivalent stress at peak pressure in the longitudinal cross section where the maximum values were found. The high stress was distributed on the cap and near the interfaces between the medial wall and lipid core base. Maximum equivalent stresses on the cap are shown in Table 1, and the values of maximum cap stress increased as the asymmetry increased. The location of maximum cap stress was near the throat for the symmetric model, and it moved to the proximal cap for the asymmetric models (see arrow heads in the Fig. 4). The point of the maximum cap stress located on the proximal throat region, where plaque rupture, was frequently observed. Because the cap stress, which is a potential physical factor that contributes to plaque rupture, increased as the asymmetry increased, we suspect that asymmetric plaque geometry could increase the danger of plaque rupture. This observation also agreed with clinical findings that upstream ruptured plaques exhibited a strongly pronounced longitudinal asymmetry (Cicha et al., 2011).

Table1. Stress and strain at peak pressure and wall shear stress at peak flow for different models

\begin{tabular}{l|c|c|c}
\hline \hline & Stress $(\mathrm{kPa})$ & Strain & Wall Shear Stress (Pa) \\
\hline Sym & 34.5 & 0.081 & 52.9 \\
Asym 1 & 69.6 & 0.104 & 60.5 \\
Asym 2 & 77.1 & 0.098 & 72.5 \\
Asym 3 & 144.4 & 0.105 & 58.9 \\
\hline
\end{tabular}



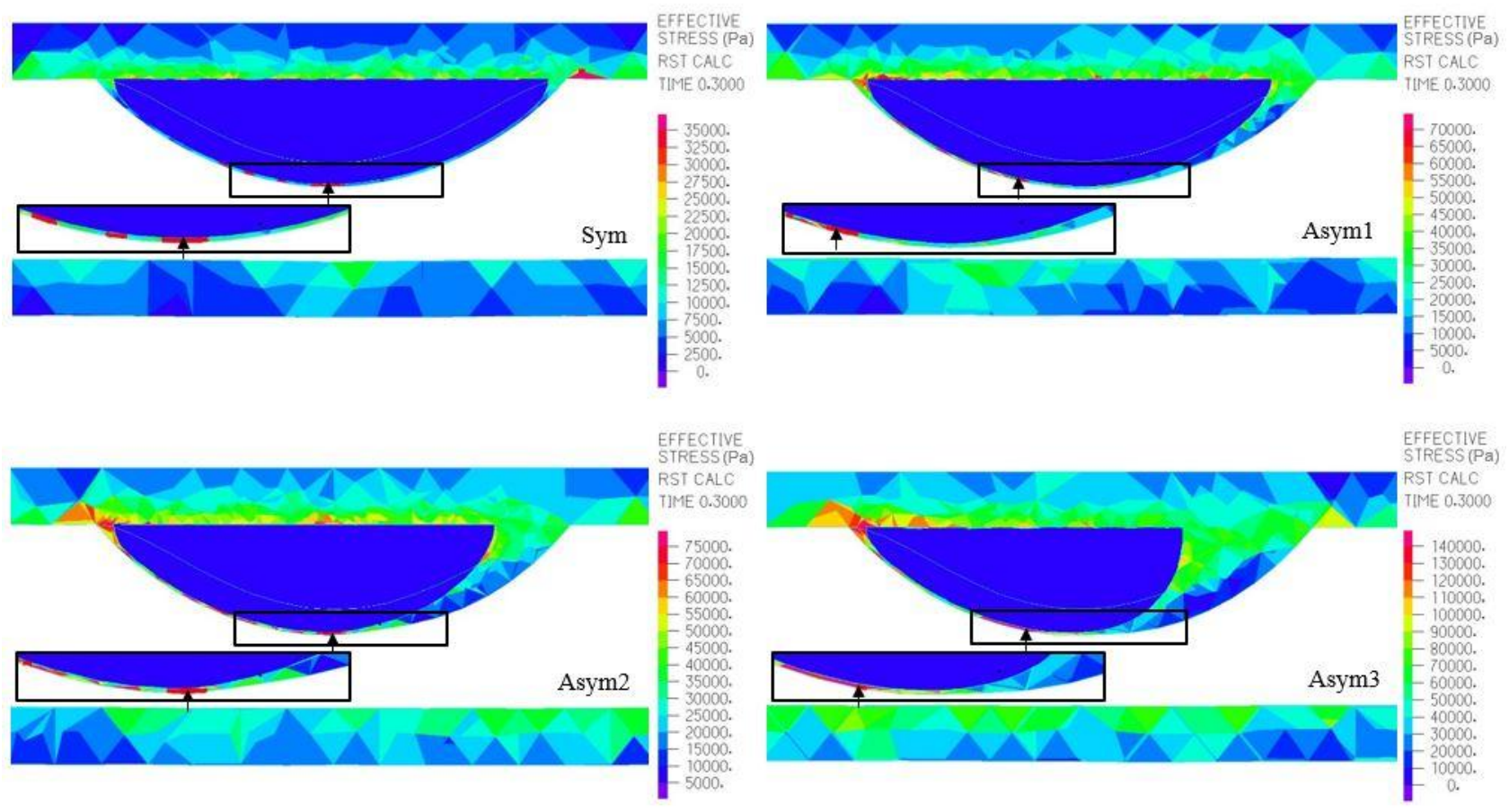

Fig. 4 Equivalent stress contour in the longitudinal cross section at peak pressure (the arrow head denotes max stress location).The maximum cap stress increased as the asymmetry increased, and the location of it moved to the proximal cap for the asymmetric models.
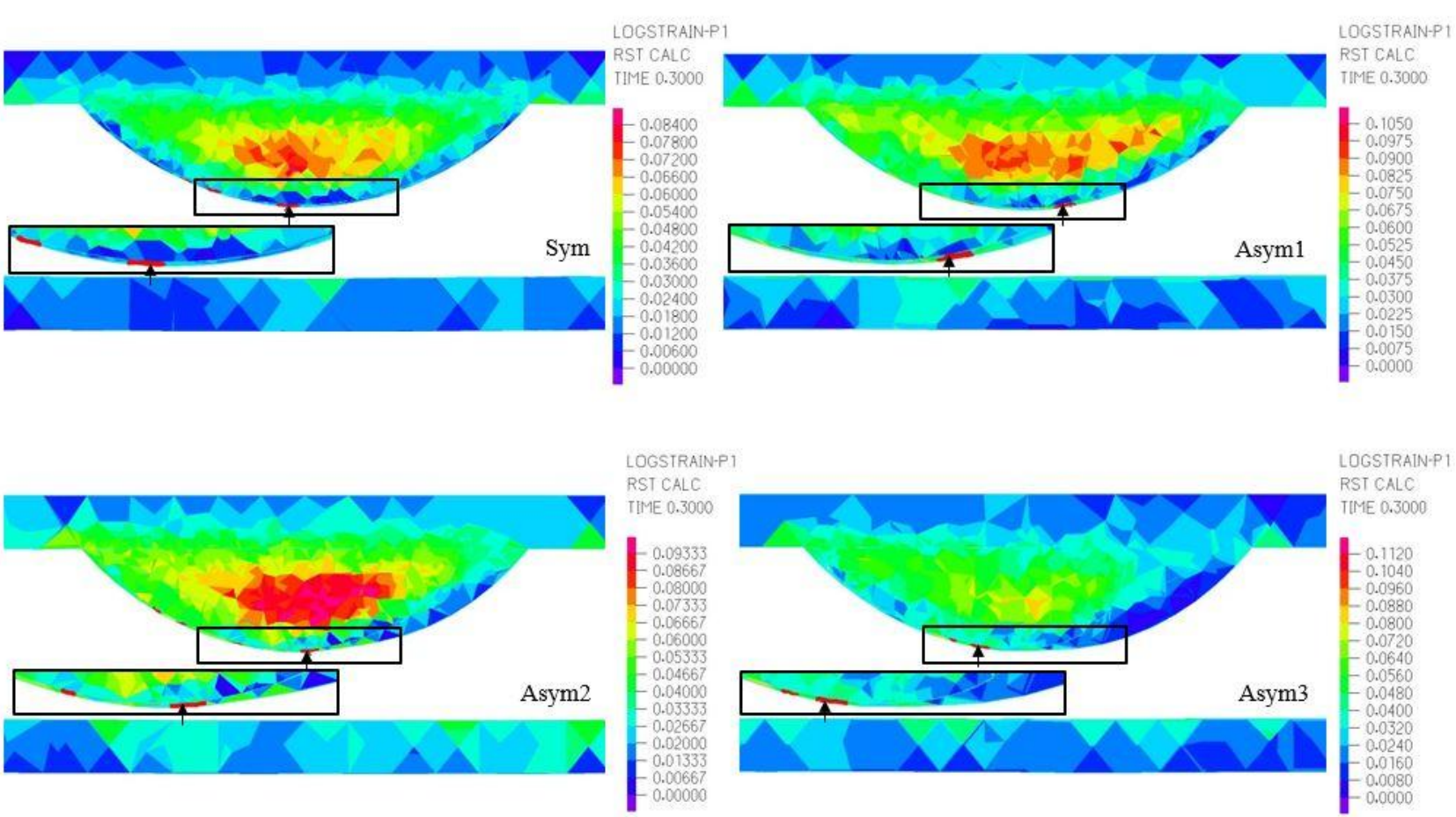

Fig. 5 Strain contour at peak pressure in the longitudinal cross section (the arrow head denotes max strain location). The high strain region coincided with the lipid core region and the fibrous cap. The maximum cap strains in the asymmetric models were higher compared to those in the symmetric model. 

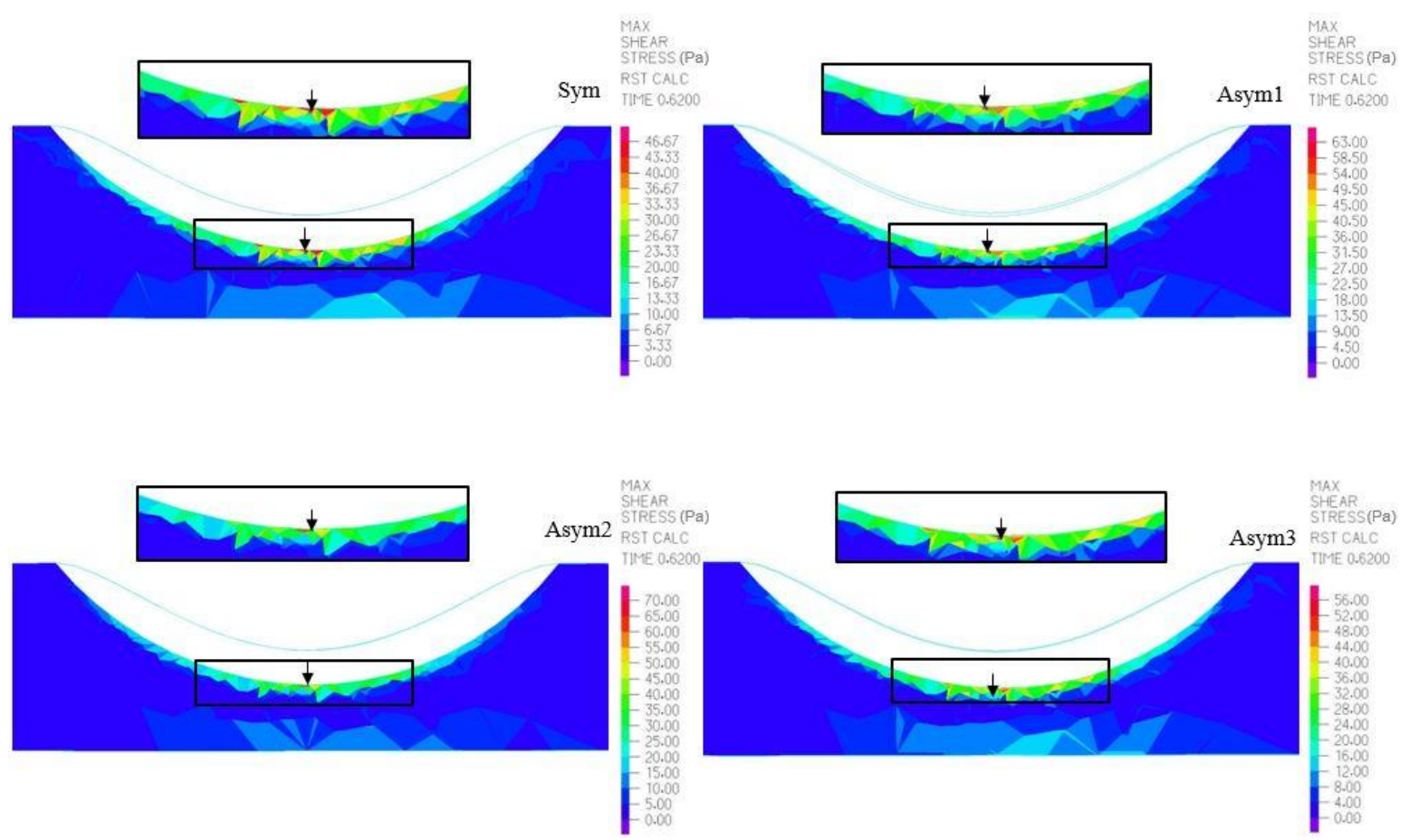

Fig. 6 Hemodynamic shear stress contours in the longitudinal cross section at peak flow (the arrow head denotes max WSS locations). The maximum WSSs were observed near the throat of the stenosis.

The wall strain was also compared for the different plaque models. Stains were higher in the lipid core regions and the cap tissues. The maximum strains in the fibrous cap (cap strains) in the asymmetric models were higher compared to those in the symmetric model, but they did not change noticeably for the different asymmetric models (Table 1). The high strain region coincided with the lipid core region, and it decreased as the size of a lipid core decreased for asymmetry models, as shown in Fig. 5. Furthermore, higher cap strains were shown in the proximal cap region. These strains may cause more vulnerabilities because of a strong positive correlation between strain and macrophage content (Gijsen et al., 2013). Compared to other models, asymmetry model 3, which has a smaller lipid core size and a thicker distal cap, had a lower cap strain in the distal cap region.

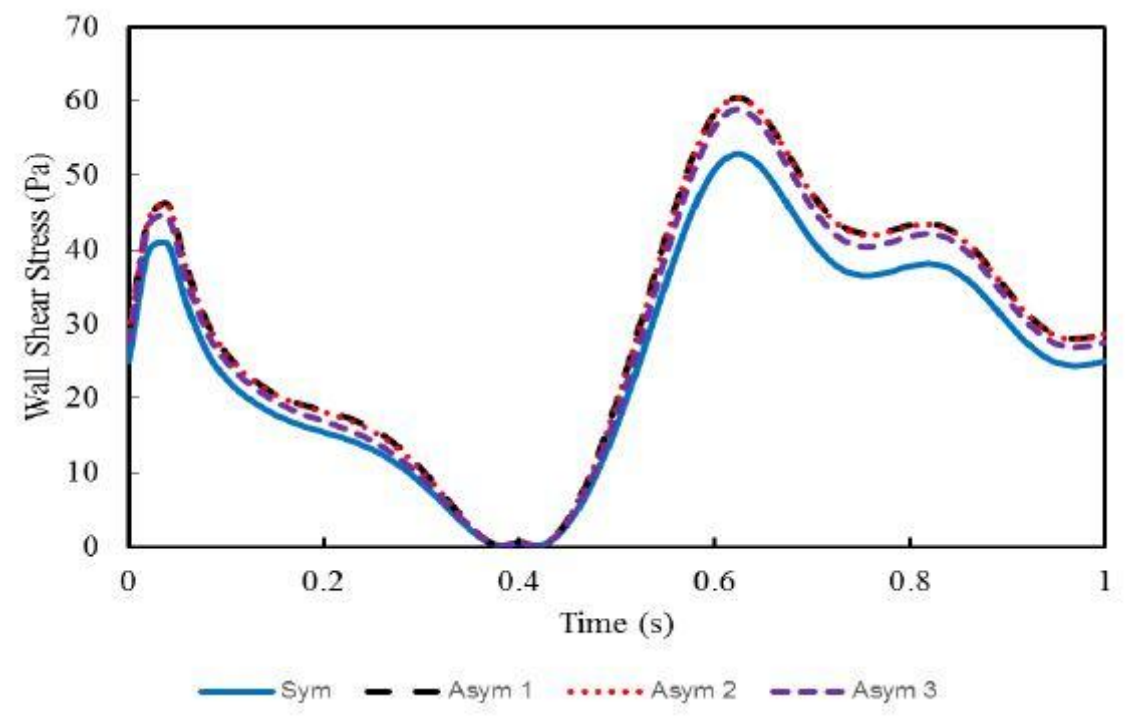

Fig. 7 Temporal variation of wall shear stresses at the throat. The maximum WSSs in the asymmetry models at peak flow were higher than those in the symmetry model. 
Hemodynamic shear stress contours at peak flow are shown in Fig. 6. The wall shear stress (WSS) due to fluid motion is high near the throat of the stenosis, which is where maximum WSSs were observed. WSSs were higher in the distal sites than in the proximal sites of the throat, but they were lower near the distal end of the stenosis. The maximum WSSs at the throat in the asymmetry models at peak flow were higher than those in the symmetry model (Fig. 7). The temporal variation of the WSS was also higher in the asymmetry models.

The maximum and temporal mean wall shear stresses along the stenotic region are shown in Fig. 8. Both the maximum and mean WSSs were highest near the throat for all models, and they were higher in the mid-distal sites than in the mid proximal sites for all models. The WSSs were lower in the symmetric models than in the asymmetry models, but the degree of asymmetry (skewness of core distribution) did not significantly affect WSS distribution. The spatial gradient of WSS (the slope of shear stress distribution curve) was higher in the proximal throat compared to changes in WSS decrease in the distal throat. The spatial gradient of WSS was higher in the asymmetry models than in the symmetry model. The temporal and spatial gradients of WSS affect the plaque progression and wall thickening (Soulis et al., 2014), and high peak and spatial gradients of WSS stimulate matrix degrading proteins, which induce wall weakness (Dolan et al.,2013, Li et al., 2009). Because the asymmetric models showed higher WSS temporal and spatial gradients than the symmetric models, we speculate that hemodynamic stresses in asymmetric lipid core distribution may promote thinning and weakening of the plaque wall. Figure 9 shows the maximum and temporal mean pressure distributions along the stenotic region. The pressure drop and pressure gradient across the stenotic region were higher in the asymmetric models than in the symmetric models. The increased pressure drop across the stenosis may increase plaque vulnerability because of larger pressure drag force on the plaque (Li et al., 2009). The pressure distribution was not significantly influenced by its degree of asymmetry.
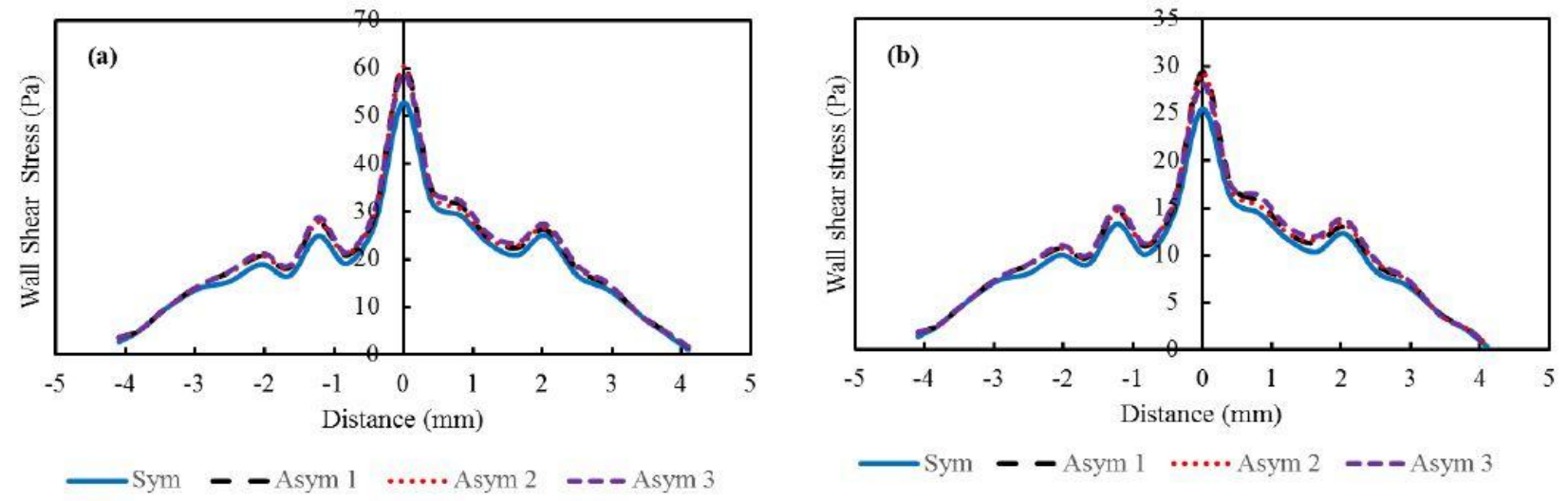

Fig. 8 Maximum wall shear stress (a) and mean wall shear stress (b) distributions along the stenotic region. They were lower in the symmetric models than in the asymmetry models. Abscissa denotes the distance from the throat.
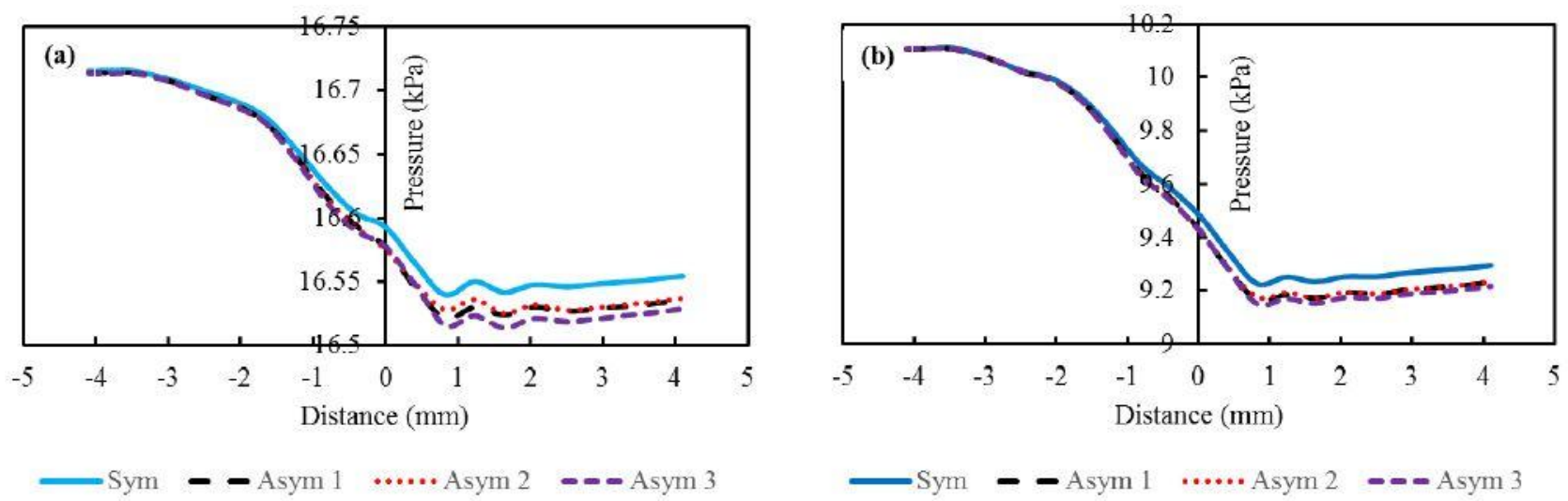

Fig. 9 Maximum pressure (a) and mean pressure (b) distributions along the stenotic region. The pressure drop and pressure gradient across the stenotic region were higher in the asymmetric models than in the symmetric models. Abscissa denotes the distance from the throat. 
Stenotic arterial geometry provides the endothelium with varying hemodynamic environment, which affects vascular cellular functions. The upstream side of the stenosis is characterized by steep increase of WSS while a flow separation and recirculation, and the low and oscillating WSS are observed on the downstream of the stenosis. The different lipid core distribution might change the luminal geometry in the stenotic region due to wall deformation caused by fluid motion. FSI simulation was performed, and the lumen geometry change due to different lipid core distribution was barely noticeable. But, noticeable differences in the WSS gradients and pressure were found between symmetric and asymmetric models. Because the WSS and pressure gradient affected wall weakening and dragging force on the plaque, asymmetric lipid core distribution might affect the plaque stability.

Though the effects of asymmetric distribution of the lipid core on the mechanical parameters were explored in this computational study, there were many limitations that should be improved in further studies. The first limitation is related the idealized plaque and lumen geometries. The patient specific models which were constructed from the ultrasound and MRI images would provide more realistic vessel geometries, but sufficient number of simulations should be performed to draw meaningful results accounting for the individual geometrical differences of patients. Also, the small size wall tissues such as calcium deposits and thrombi were not considered, which might affect local stress concentration. The second limitation is related to the modeling of the material properties of plaque wall and blood. Anisotropic and viscoelastic properties in the arterial wall tissues were not modelled, because they were less serious in the plaque tissues and lipid core (Yuan et al., 2015). Non-Newtonian blood viscosity and turbulence were not modelled, because flow characteristic would not affect the wall stress a lot via FSI. Another limitation is related to wall stress calculations. Residual stress, arterial curvature and cycle bending due to cardiac motions were not considered, which might affect the wall stress a lot (Tang et al., 2009). These limitations should be kept in mind when interpreting our simulation results. Nevertheless, major results from this computational study would provide meaning insight in analyzing plaque wall stability.

\section{Conclusion}

In this study, computational analysis incorporating FSI was performed in order to study the effect of the longitudinal asymmetric distribution of the lipid core on arterial wall mechanics and hemodynamics. The values of maximum cap stress increased, and its location moved toward the proximal cap as asymmetry increased. The maximum strains in the asymmetric models were also higher compared to those in the symmetric model. Because high wall stress and wall strain are positively correlated with plaque rupture, longitudinal asymmetric distribution of the lipid core may increase the vulnerability of the plaque.

The hemodynamic WSS did not change much with longitudinal asymmetry, because the luminal geometry varied only slightly in the asymmetry models. However, the maximum WSS and its spatial gradient, which were believed to be positively related to the endothelium denudation and degradation, were higher in the asymmetry models than in the symmetry model. Furthermore, the pressure drop and pressure gradient across the stenosis were higher in the asymmetry models than in the symmetry model. Therefore, higher wall stress and strain, and increased WSS, pressure drops, and gradients of them in asymmetric plaques may provide a more unfavorable biomechanical environment for plaque stability.

\section{Acknowledgments}

This work was supported by the Research Fund (NRF-2014R1A2A1A11051357). 


\section{References}

Akyildiz, A. C., Speelman, L., vanBrummelen, H., Gutiérrez, M. A., Virmani, R., van der Lugt, A., van der Steen, A. F., Wentzel, J. J. and Gijsen, F. J., Effects of intima stiffness and plaque morphology on peak cap stress, Biomed. Eng. Online, Vol.10, (2011), 25.

Bathe, K. J., Finite Element Procedures (1996), Prentice-Hall, Englewood Cliffs, NJ.

Bathe, K. J., Theory and Modeling Guide, Vols. I \& II: ADINA and ADINA-F (2002), ADINA R \&D, Inc., Watertown, MA.

Burke, A. P., Farb, A., Malcom, G. T., Liang, Y. H., Smialek, J. and Virmani, R., Coronary risk factors and plaque morphology in men with coronary disease who died suddenly, N Eng J Med, Vol.336, No.18 (1997), pp.1276-1282.

Cicha, I., Wörner, A., Urschel, K., Beronov, K., Goppelt-Struebe, M., Verhoeven, E., Werner Daniel, W. G. and Garlichs, C. D., Carotid Plaque Vulnerability A Positive Feedback Between Hemodynamic and Biochemical Mechanisms, Stroke, Vol.42, No.12 (2011), pp.3502-3510.

Dirksen, M. T., van der Wal, A. C., van de rBerg, F. M., van der Loos, C. M. and Becker, A. E., Distribution of inflammatory cells in atherosclerotic plaques relates to the direction of flow, Circulation, Vol.98, No.19 (1998), pp. $2000-2003$.

Dolan J. M, Kolega J. and Meng, H., High Wall Shear Stress and Spatial Gradients in Vascular Pathology: A Review, Ann Biomed Eng, Vol.41, No.7 (2013), pp.1411-1427.

Gijsen, F. J., Wentzel, J. J., Thury, A., Mastik, F., Schaar, J. A., Schuurbiers, J. C., Slager, C. J., vanderGiessen, W. J., deFeyter, P. J., vanderSteen, A. F. and Serruys, P. W., Strain distribution over plaques in human coronary arteries relates to shear stress, American Journal of Physiology-Heart and Circulatory Physiology, Vol.295, No.4 (2008), H1608-H1614.

Gijsen, F., vanderGiessen, A., vanderSteen, A. and Wentzel, J., Shear stress and advanced atherosclerosis in human coronary arteries, Journal of Biomechanics, Vol.46, No.2 (2013), pp.240-247.

Kelly-Arnold, A., Maldonado, N., Laudier, D., Aikawa, E., Cardoso, L. and Weinbaum, S., Revised microcalcification hypothesis for fibrous cap rupture in human coronary arteries, Proc.Natl.Acad.Sci. USA, Vol.110, No.26 (2013), pp.10741-10746.

Kural, M. H., Cai, M., Tang, D., Gwyther, T., Zheng, J. and Billiar, K. L., Planar biaxial characterization of diseased human coronary and carotid arteries for computational modeling, J Biomech, Vol.45, No.5 (2012), pp.790-798.

Li, Z. Y., Taviani, V., Tang, T., Sadat, U., Young, V., Patterson, A., Graves, M. and Gillard, J. H., The mechanical triggers of plaque rupture: shear stress vs pressure gradient, Br J Radiol, Vol.29, No.1 (2009), S39-45.

Libby, P., Mechanisms of acute coronary syndromes, N Engl J Med, Vol.369, No.9 (2013), pp.883-884.

Nieuwstadt, H. A., Akyildiz, A. C., Speelman, L., Virmani, R., vanderLugt, A., vanderSteen, A. F., Wentzel, J. J. and Gijsen, F. J., The influence of axial image resolution on atherosclerotic plaque stress computations, J.Biomech, Vol.46, No.4 (2013), pp.689-695.

Ohayon, J., Finet, G., Treyve, F., Rioufol, G. and Dubreuil, O., A three-dimensional finite element analysis of stress distribution in a coronary atherosclerotic plaque: In-vivo prediction of plaque rupture location, Biomechanics Applied to Computer Assisted Surgery, Vol.37, No.2 (2005), pp.225-241.

Ohayon, J., Finet, G., Gharib, A. M., Herzka, D. A., Tracqui, P., Heroux, J., Rioufol, G., Kotys, M. S., Elagha, A. and Pettigrew, R. I., Necrotic core thickness and positive arterial remodeling index: emergent biomechanical factors for evaluating the risk of plaque rupture, Am. J. Physiol. Heart Circ. Physiol, Vol.295, No.2 (2008), H717-H727.

Peiffer, V., Sherwin, S. J. and Weinberg, P. D., Does low and oscillatory wall shear stress correlate spatially with early atherosclerosis? A systematic review, Cardiovasc Res, Vol.99, No.2 (2013), pp.242-250.

Rambhia S. H., Liang, X., Xenos, M., Alemu, Y., Maldonado, N., Kelly, A., Chakraborti, S., Weinbaum, S., Cardoso, L., Einav, S. and Bluestein, D., Microcalcifications increase coronary vulnerable plaque rupture potential: a patient-based micro-CT fluid-structure interaction study, Ann Biomed Eng, Vol.40, No.7 (2012), pp.1443-1454.

Slager, C. J., Wentzel, J. J., Gijsen, F. J., Thury, A., vanderWal, A. C., Schaar, J. A. and Serruys, W. P., The role of shear stress in the destabilization of vulnerable plaques and related therapeutic implications, Nat Clin Pract Cardiovasc Med, Vol.2, No.9 (2005), pp.456-464.

Soulis, J. V., Fytanidis, D. K., Seralidou, K. V. and Giannoglou G. D., Wall shear stress oscillation and its gradient in 
the normal left coronary artery tree bifurcations, Hippokratia, Vol.18, No.1 (2014), pp.12-16.

Tang, D., Yang, C., Kobayashi, S., Zheng, J., Woodard, P. K., Teng, Z., Billiar, K., Bach, R. and Ku, D. N., 3D MRI-based anisotropic FSI models with cyclic bending for human coronary atherosclerotic plaque mechanical analysis, J Biomech Eng, Vol.131, No.6 (2009), p.061010.

Tang, D., Kamm, R. D., Yang, C., Zheng, J., Canton, G., Bach, R. and Maehara, A., Image-based modeling for better understanding and assessment of atherosclerotic plaque progression and vulnerability: Data, modeling, validation, uncertainty and predictions, Journal of biomechanics, Vol47, No.4 (2014), pp.834-846.

Wang, L., Zheng, J., Maehara, A., Yang, C., Billiar, K. L., Wu, Z. and et al., Morphological and Stress Vulnerability Indices for Human Coronary Plaques and Their Correlations with Cap Thickness and Lipid Percent: An IVUS-Based Fluid-Structure Interaction Multi-patient Study, PLoS Comput Biol, Vol.11, No.12 (2015) e1004652.

Yang, C., Canton, G., Yuan, C., Ferguson, M., Hatsukami, T. S. and Tang, D., Advanced human carotid plaque progression correlates positively with flow shear stress using follow-up scan data: an in vivo MRI multi-patient 3D FSI study, Journal of biomechanics, Vol.43, No.13 (2010), pp.2530-2538.

Yilmaz, A., Lipfert, B., Cicha, I., Schubert, K., Klein, M., Raithel, D., Daniel, W. G. and Garlichs, C. D., Accumulation of immune cells and high expression of chemokines/ chemokine receptors in the upstream shoulder of atherosclerotic carotid plaques, Exp Mol Pathol, Vol.82, No.3 (2007) pp.245-255.

Yuan, J., Teng, Z., Feng, J., Zhang, Y., Brown, A. J., Gillard, J. H. and Lu, Q., Influence of material property variability on the mechanical behaviour of carotid atherosclerotic plaques: A 3D fluid-structure interaction analysis, International journal for numerical methods in biomedical engineering, Vol.31 (2015) 8.

Zareh, M., Fradet, G., Naser, G. and Mohammadi, H., Are two-dimensional images sufficient to assess the atherosclerotic plaque vulnerability: a viscoelastic and anisotropic finite element model, Cardio Vasc Syst, Vol.3 (2015), 3 . 